\title{
Distributed-Mass Micromachined Gyroscopes for Enhanced Mode-Decoupling
}

\author{
Cenk Acar \\ Microsystems Laboratory \\ Mechanical and Aerospace Engineering Dept. \\ University of California at Irvine \\ Irvine, CA, USA \\ cacar@uci.edu
}

\author{
Andrei M. Shkel \\ Microsystems Laboratory \\ Mechanical and Aerospace Engineering Dept. \\ University of California at Irvine \\ Irvine, CA, USA \\ ashkel@uci.edu
}

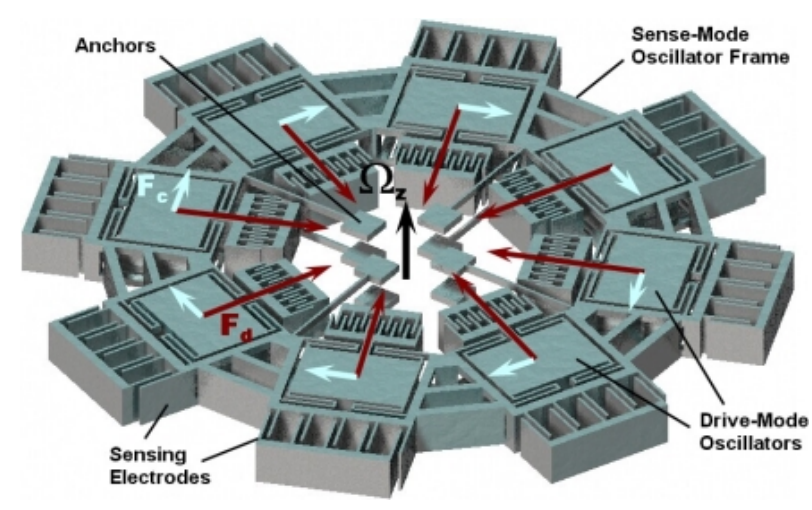

Figure 1. Conceptual illustration of the Distributed-Mass Gyroscope (DMG).

sense directions. The proof mass is sustained in resonance in the drive direction, and in the presence of an angular rotation, the Coriolis force proportional to the input angular rate is induced, exciting the proof mass in the sense direction. To achieve high sensitivity, the drive and the sense resonant frequencies are typically designed and tuned to match to a certain degree, and the device is controlled to operate at or near the peak of the response curve $[1,4]$.

The drive and sense mode matching requirement in vibratory gyroscopes renders the system response very sensitive to variations in system parameters due to fabrication imperfections and fluctuations in operating conditions, which shift the drive or sense resonant frequencies [6]. For the devices packaged in vacuum to enhance the sensitivity, the bandwidth of the resonance peaks is extremely narrow; leading to much tighter modematching requirements. Extensive research has been focused on design of symmetric drive and sense-mode suspensions for mode-matching and minimizing temperature dependence $[13,15]$. However, especially for lightly-damped devices, the mode-matching requirement is well beyond fabrication tolerances; and none of the symmetric designs can provide the required degree of mode-matching without feedback control $[4,5]$.

Furthermore, as the modes are matched closer, the mechanical interference between the modes becomes more significant, resulting in operation instability and drift. 
In order to suppress coupled oscillation and drift and to minimize the resulting zero-rate drift, various devices have been reported employing independent suspension beams for the drive and sense modes $[8,9,10,11,13]$. The approach of decoupling drive and sense modes led to the first integrated commercial MEMS gyroscopes produced by Analog Devices [14]. The DistributedMass Gyroscope (DMG) concept also aims to decouple the drive and sense modes, with the difference of employing multiple linear drive-modes completely decoupled from the rotational sense-mode; while providing a wide-band robust drive-mode.

\section{GYROSCOPE STRUCTURE AND PRINCIPLE OF OPERATION}

We propose a novel micromachined gyroscope system with multi-directional linear drive-mode and a rotational sense-mode to minimize quadrature error, eliminate effects of directional residual stresses, and effectively decouple the drive and sense modes.

The design concept is based on forming multiple drivemode oscillators, distributed symmetrically around the center of a supporting frame. The distributed drivemode oscillators are driven in-phase along the axes normal to the tangents of the supporting frame, and constrained in the tangential direction with respect to the supporting frame. In the presence of an angular rotation rate about the $\mathrm{z}$-axis, the Coriolis forces are induced on each proof mass orthogonal to each drivemode oscillation direction (Figure 1). Thus, each of the induced Coriolis force vectors lie in the tangential direction, combining to generate a resultant torque on the supporting frame. The net Coriolis torque excites the supporting frame into torsional oscillations about the z-axis, which are detected by sensing capacitors for angular rate measurement.

The multi-directional and axi-symmetric nature of the drive-mode oscillators has several benefits over a conventional gyroscope design. Firstly, since the drive forces applied to the drive-mode oscillators cancel out in all directions due to the radial symmetry, the net driving force on the structure reduces to zero. The symmetry of the design is assured in the fabrication process, where the complete structure is defined lithographically in one mask, eliminating the possibility of mask misalignment. Furthermore, the sensing electrodes attached to the supporting frame do not deflect due to the drive-mode oscillations. Secondly, instability and drift due to mechanical coupling between the drive and sense modes is minimized, since the structure allows complete decoupling of multi-directional linear drive-mode and the rotational sense-mode. Thus, zero-rate-output and quadrature error are suppressed effectively in the presence of structural imperfections. The symmetry of the drive-mode oscillator structure about several axes also

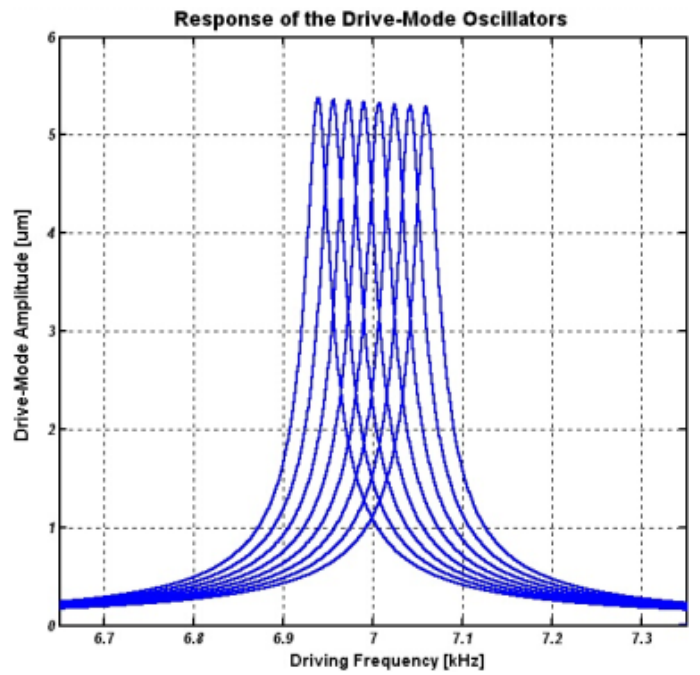

(a)

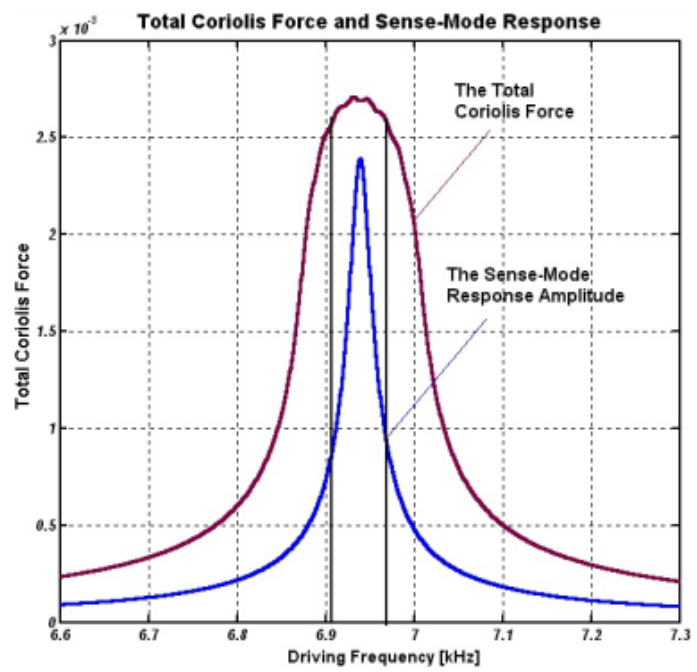

(b)

Figure 2. (a) The frequency responses of the distributed drivemode oscillators. (b) The frequency spectrum of the total Coriolis torque generated by the distributed drive-mode oscillators.

cancels the effects of directional residual stresses, and elastic anisotropy of the structural material.

\section{The Coriolis Response}

In the proposed approach, the distributed drive-mode oscillators are driven in-phase towards the center, and constrained in the tangential direction with respect to the supporting frame. The constrained dynamics of each proof-mass along the associated drive axis with respect to the supporting frame reduces to:

$$
m_{i} \ddot{x}_{i}+c_{x} \dot{x}_{i}+k_{x} x_{i}=F_{d}+2 m_{i} \Omega_{z} \dot{y}_{i}
$$

where $m_{i}$ is the $i^{t h}$ proof-mass, and $x_{i}$ it the drivemode oscillation amplitude of the $i^{t h}$ mass. Thus, in the presence of an angular rotation rate about the $\mathrm{z}-$ axis, the Coriolis forces, which are proportional to drive 
direction oscillation amplitudes, induced on each proof mass are:

$$
F_{c i}=2 m_{i} \Omega_{z} \dot{x_{i}}
$$

The rotation-induced Coriolis forces are orthogonal to each drive-mode oscillation direction. Thus, each of the induced Coriolis force vectors lie in the tangential direction, combining to form a resultant torque on the supporting frame. The net Coriolis torque generated as the combination of each Coriolis force becomes:

$$
\vec{M}_{c}=\sum_{i=1}^{n} \vec{r}_{c} \times \vec{F}_{c i}=\sum_{i=1}^{n} 2 r_{c} m_{i} \Omega_{z} \dot{x_{i}} \cdot \widehat{k}
$$

where $r_{c}$ is the position vector of the oscillator center-ofmass, and $\widehat{k}$ is the unit vector in the $z$-direction. The Coriolis torque $M_{c}$ excites the supporting frame into torsional oscillations about the $\mathrm{z}$-axis, which are detected by sensing capacitors for angular rate measurement. Thus, the equation of motion of the supporting frame in the sense-direction is

$$
I_{z} \ddot{\phi}+D_{z} \dot{\phi}+K_{z} \phi=M_{c},
$$

where $\phi$ is the torsional deflection of the supporting frame, $I_{z}$ denotes the moment of inertia of the supporting frame combined with the proof masses, $D_{z}$ is the sense-mode rotational damping ratio, and $K_{z}$ is the torsional stiffness of the suspension structure.

\section{WIDE-BANDWIDTH OPERATION FOR IMPROVING ROBUSTNESS}

The most prominent advantage of the design concept is the capability to provide a wide-bandwidth operation region in the drive-mode frequency response. If each drive-mode oscillator proof-mass is designed to incrementally increase by $\Delta m$ such that

$$
m_{i}=m_{0}+i \Delta m,
$$

then the resonance frequencies of each drive-mode oscillator will be incrementally spaced (Figure 2a). Since the tangential Coriolis forces induced on each proof mass jointly generate a resultant torque on the supporting frame, a constant total Coriolis torque is achieved over a wide range of driving frequencies (Figure $2 \mathrm{~b}$ ). The device is nominally operated in the flat region of the Coriolis torque frequency response. Thus, fluctuation in system parameters that shift oscillator resonance frequencies will not result in a significant change in the total Coriolis torque through the operation frequency band. If the sense-mode resonance frequency is designed to be accommodated in the same frequency band (Figure $2 \mathrm{~b}$ ), the requirement on the degree of mode-matching is relaxed, and robustness against structural and thermal parameter fluctuations is achieved.

It should be noticed that the resonance frequency separation of the oscillators are dictated by the bandwidth of the response, which is determined by damping. In order to obtain a flat operation region in the drive-mode,

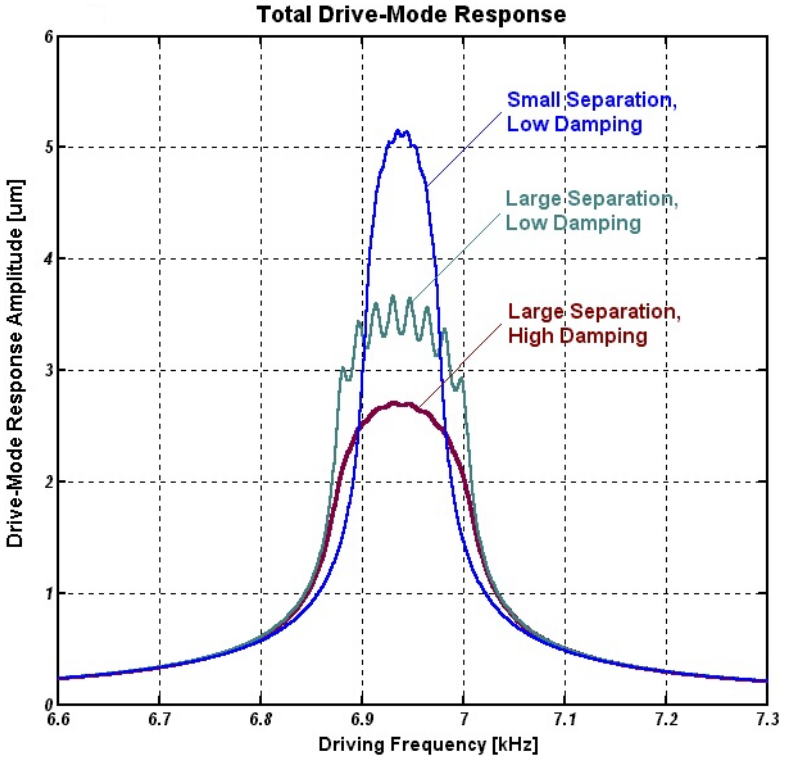

Figure 3. The effect of damping and resonance frequency separation on the drive-mode response.

the frequency separation should be less that $10 \%$ of the bandwidth. If the separation of frequencies is large for low damping resonators, spacing of the resonance peaks become significant in the response (Figure 3 ), and the flat operation region will not be achieved in the drivemode response.

\section{Sensitivity and Robustness Analyses}

The proposed design approach allows to widen the operation frequency range of the gyroscope drive-mode to achieve improved robustness, while sacrificing the response amplitude. The optimal compromise between amplitude of the response and bandwidth (effecting sensitivity and robustness, respectively) can be obtained by selecting the frequency increments of the drive-mode oscillators.

As a numerical example, the response of a device consisting of 8 drive-mode oscillators with resonance frequencies located at 6.87 to $6.99 \mathrm{kHz}$ and a frequency spacing of $15 \mathrm{~Hz}$ will be analyzed. For $1^{\circ} / \mathrm{sec}$ input angular rate and a $\mathrm{Q}$ factor of 100 in the drive and sense modes, the supporting frame of the distributed-mass gyroscope will have an amplitude of response equal to $2.8 \times 10^{-3} \mu m$ at the sensing electrodes. If the frequency spacing of the drive-mode oscillators is decreased from $15 \mathrm{~Hz}$ to $10 \mathrm{~Hz}$, the amplitude of the response in the sense direction will increase from $2.8 \times 10^{-3} \mu m$ to $3.9 \times$ $10^{-3} \mu \mathrm{m}$; while the response bandwidth will decrease from $200 \mathrm{~Hz}$ to $140 \mathrm{~Hz}$, which is still over an order of magnitude larger than the bandwidth of a single-mass conventional gyroscope. The bandwidth can be further widened by increasing the number of oscillators (Figure 4). Thus, the design concept provides more freedom in 


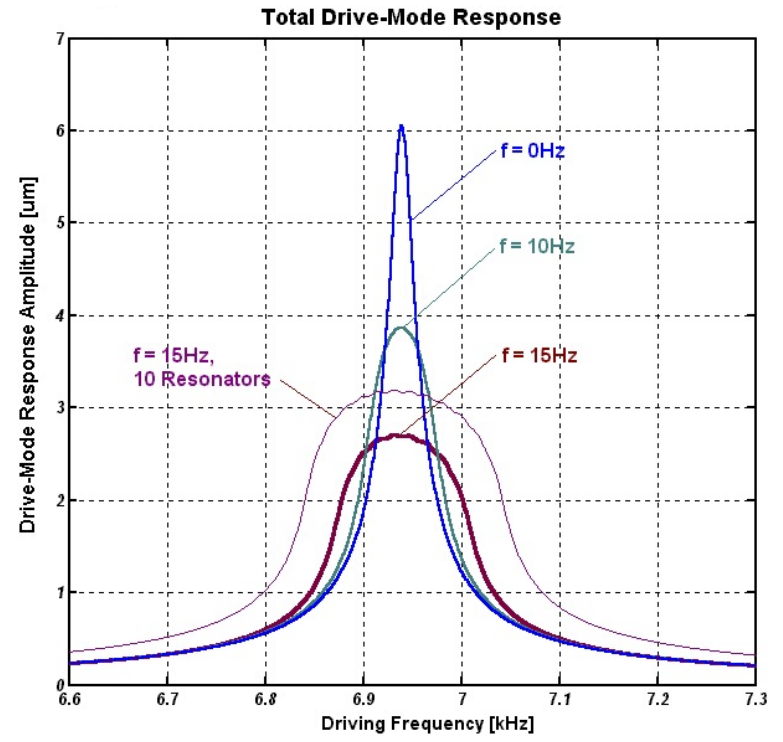

Figure 4. The effect frequency separation on the response gain and bandwidth (effecting sensitivity and robustness, respectively). The gain is maximized for zero frequency separation, and the overall bandwidth increases proportional to spacing.

defining trade-offs between gain of the response (higher sensitivity) and the system bandwidth (increased robustness), typically guided by application requirements.

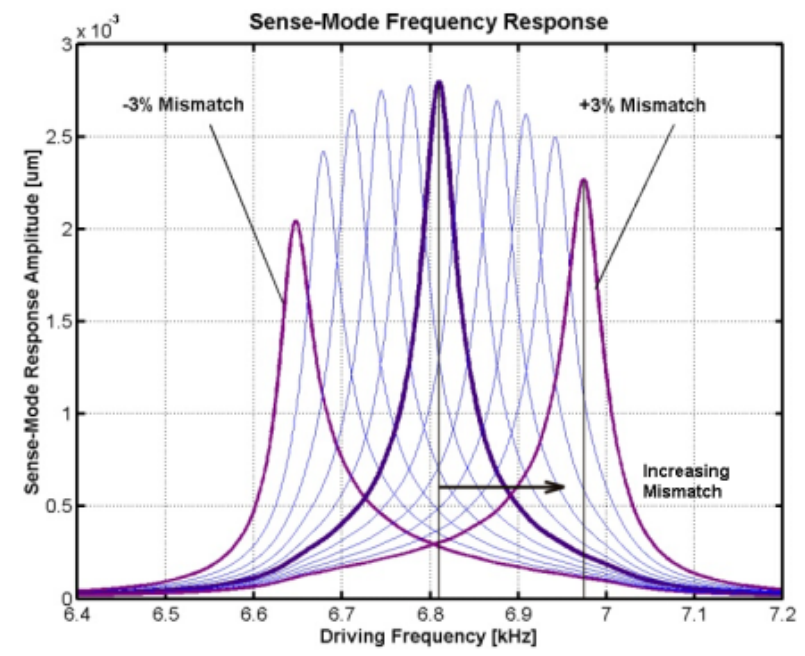

Figure 5. The results of frequency mismatch on the overall system response. A mismatch of $3 \%$ results in only $16 \%$ error, while mismatches less than $1 \%$ have no significant effect.

Figure 5 illustrates the case of a potential shift in the sense-mode resonance frequency, e.g. due to temperature fluctuations, residual stresses, or fabrication variations. It is observed that the response amplitude is sustained at a constant value to a great extent without requiring feedback control or active tuning of resonance frequencies. For example, a $3 \%$ shift in the sense-mode resonance frequency results in only $16 \%$ error in the response amplitude, while mismatches less than $1 \%$ have no significant effect on the response. Without active compensation, a conventional 2-DOF gyroscope can exhibit over $60 \%$ error for the same $1 \%$ frequency shift under the same operation conditions [7].

\section{Finite Element Analysis Results}

In order to optimize the system parameters and verify the validity of the theoretical analysis assumptions, the operational modes of the system were simulated using the Finite Element Analysis package MSC Nastran/Patran. Each drive-mode mass of the analyzed prototype system is $1080 \mu m \times 510 \mu m$, suspended by four $460 \mu m \times 5 \mu m$ folded springs; yielding a resonance frequency estimation of $3.28 \mathrm{kHz}$ with $159 \mathrm{GPa}$ elastic modulus. Through FEA simulations, the drive-mode resonance frequency of the drive-mode oscillators were obtained at $3.34 \mathrm{kHz}$ (Figure 6a). The torsional sense mode resonance frequency of the structure about the sense axis was then located at $\omega_{z}=3.41 \mathrm{kHz}$ (Figure 6b) with four $769 \mu m \times 10 \mu m$ torsional suspension beams, by iteratively optimizing the beam length.

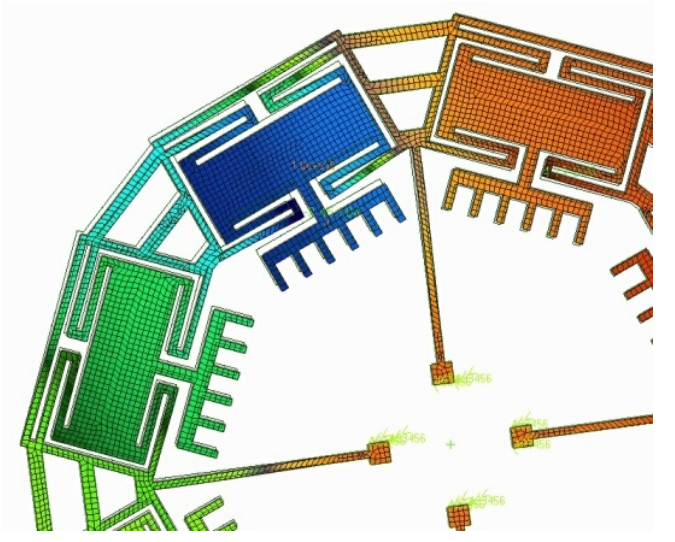

(a)

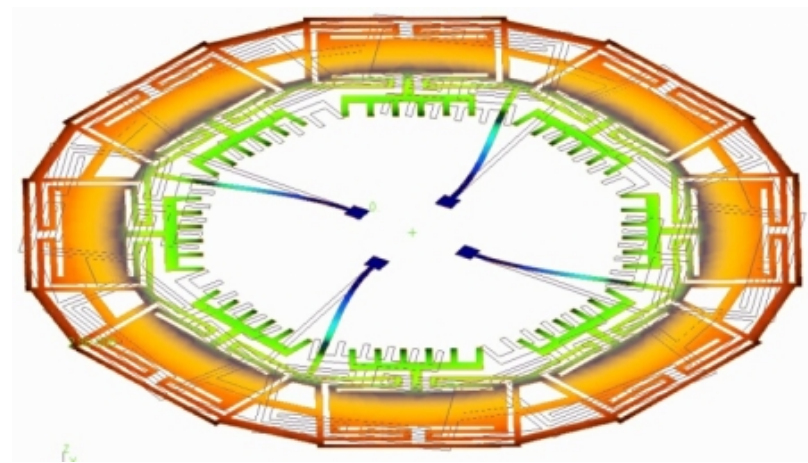

(b)

Figure 6. The FEA simulation results. (a) The linear in-plane resonance mode of the drive oscillators, obtained at $3.34 \mathrm{kHz}$. (b) The torsional sensing mode of the complete structure, optimized as $\omega_{z}=3.41 \mathrm{kHz}$. 


\section{FABRICATION OF A PROTOTYPE}

The first generation bulk-micromachined prototype gyroscopes were designed and fabricated in the UCI Integrated Nano-Research Facility. A one-mask process based on SOI (Silicon on Insulator) wafers was developed and optimized for high-aspect ratio structures. The developed process relies on deep-reactive ion etching (DRIE) through the $100 \mu \mathrm{m}$ device layer, and frontside release of the structures by etching the Oxide layer in HF solution. The process and the device design was optimized to minimize notching at the Oxide interface and excessive undercutting. In the device, $10 \mu m \times 10 \mu m$ holes were used to perforate the suspended structures, and $10 \mu \mathrm{m}$ gaps were used in the sensing and actuation electrodes. Each drive-mode oscillator was designed identically in the first generation devices.

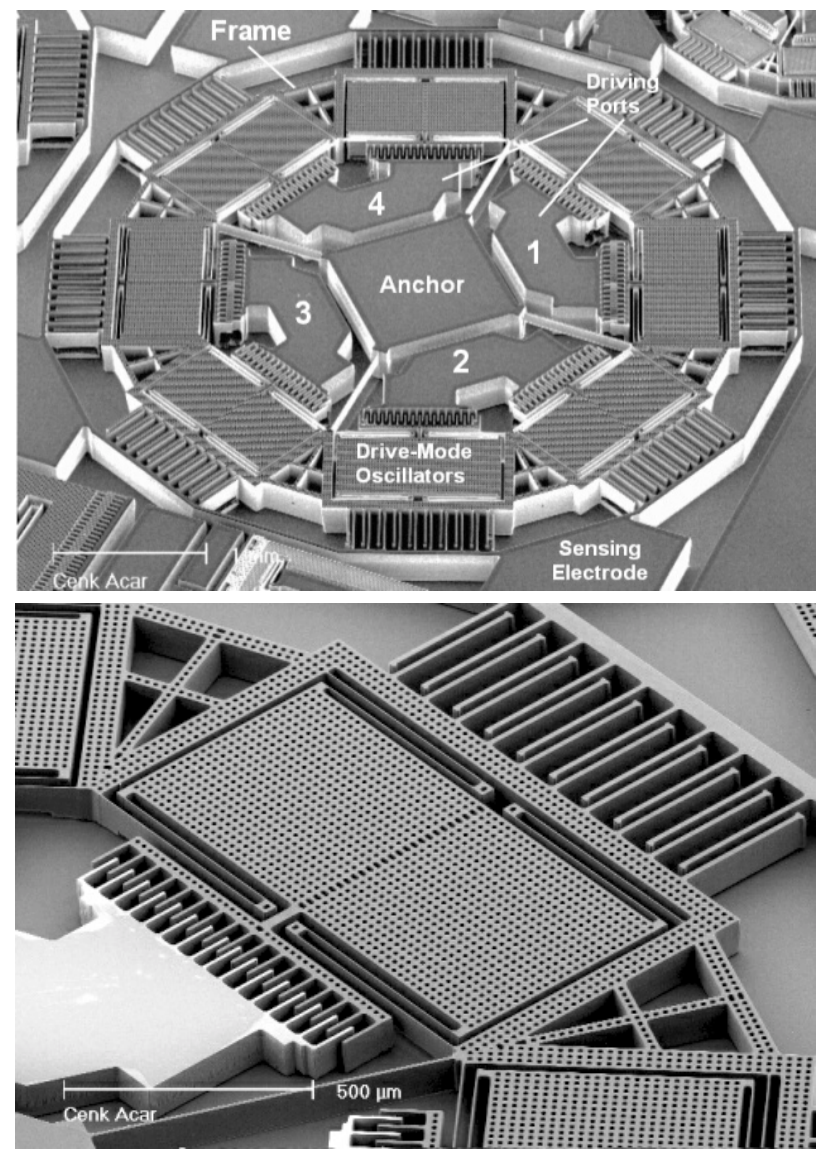

Figure 7. Scanning Electron Microscope (SEM) photograph of the characterized Distributed-Mass Micromachined Gyroscope prototype.

\section{Preliminary Experimental Results}

The linear drive-mode resonance frequencies, and the torsional sense-mode resonance frequency of the prototype gyroscope were measured in a cryogenic MMR Probe Station. The frequency response of the device was acquired under atmospheric pressure and room temperature, using off-chip transimpedance amplifiers connected to an HP Signal Analyzer in sine-sweep mode. The drive-mode frequency responses were acquired utilizing one-port actuation and detection, where a single electrode was used for both driving and sensing at the same time.

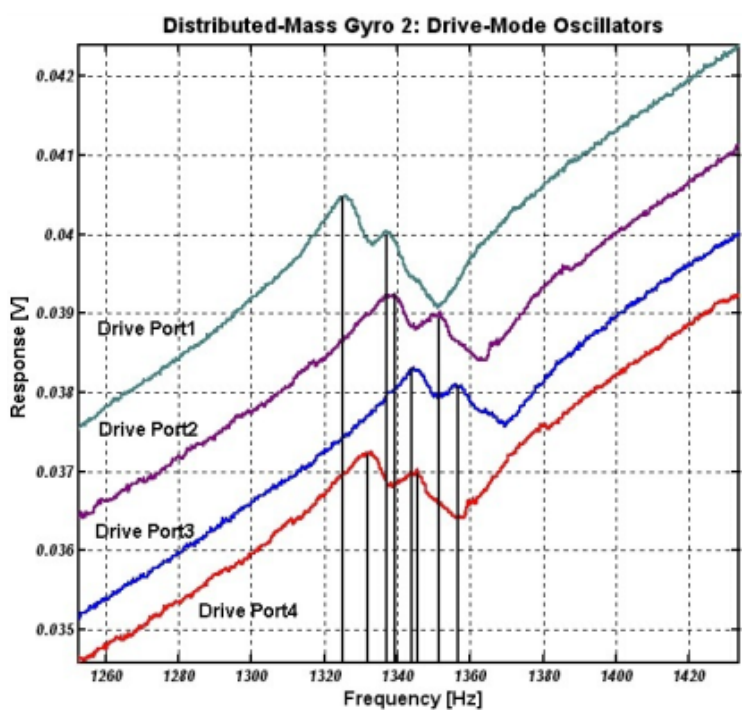

(a)

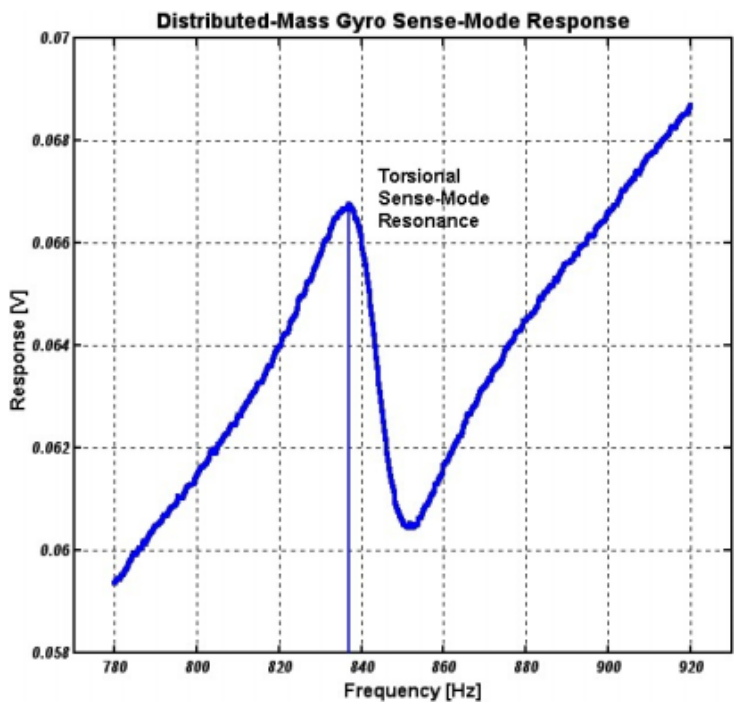

(b)

Figure 8. Experimental measurements of the frequency response: (a) The drive-mode oscillators, where each drive port is connected to two resonators; (b) The torsional sense-mode frequency response of the supporting frame.

The resonance frequencies of the drive-mode resonators were observed to be scattered between $1.324 \mathrm{kHz}$ and $1.354 \mathrm{kHz}$ within a $30 \mathrm{~Hz}$ frequency band (Figure 8a). The sense-mode resonance frequency of the frame was measured at $835 \mathrm{~Hz}$ (Figure $8 \mathrm{~b}$ ). The large deviation 
from the FEA results could be attributed to excessive lateral and vertical over-etching due to the mask resolution, and the photolithography process. In atmospheric pressure, the $\mathrm{Q}$ factor in the drive and sense modes were observed as 34 and 28, respectively. The close spacing of the drive-mode resonance frequencies allowed all of the resonators to be driven at the same time, to jointly generate a resultant Coriolis torque. The total Coriolis torque, which is estimated for $1^{\circ} / \mathrm{sec}$ input rate by summing the measured response of the four ports, was observed to provide a flat range of over $30 \mathrm{~Hz}$ (Figure 9 ). The next generation prototypes will be designed for higher drive-mode damping for wider bandwidth of the drive-mode response, and with separate ports for each resonator for individually addressable characterization.

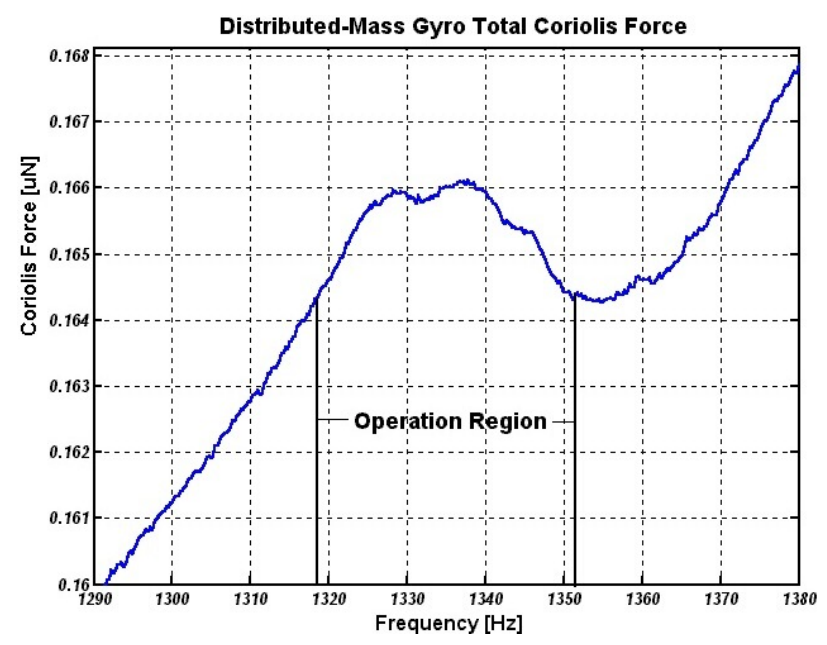

Figure 9. Experimental frequency response measurements of the total Coriolis force induced in the sense-mode for $1^{\circ} / \mathrm{sec}$ input rate, obtained by summing the measured drive-mode response of the four ports.

\section{CONCLUSION}

A novel distributed-mass micromachined gyroscope design was proposed, that is based on defining multiple drive-mode oscillators, distributed symmetrically around the center of a supporting frame. The design concept completely decouples the drive and sense modes to minimize quadrature error and zero-rate-output by forming a multi-directional linear drive-mode and a torsional sense-mode, which prevents instability and drift due to mechanical coupling to a great extent. By designing each drive-mode oscillator to have incrementally spaced resonance frequencies, a constant total Coriolis torque is achieved over a wide range of driving frequencies, leading to relaxed mode-matching requirements. Thus, the design concept is expected to provide inherently robust, low-drift and reliable MEMS gyroscopes.

\section{ACKNOWLEDGEMENTS}

This work is supported by the National Science Foundation Grant CMS-0223050. The authors gratefully acknowledge the invaluable assistance of Hung-Pin (Harrison) Chang, and $\mathrm{Vu}$ Phan in fabrication of prototypes.

\section{REFERENCES}

[1] N. Yazdi, F. Ayazi, and K. Najafi. Micromachined Inertial Sensors. Proceedings of IEEE, Vol. 86, No. 8, August 1998, pp. 1640-1658.

[2] W.A. Clark, R.T. Howe, and R. Horowitz. Surface Micromachined Z-Axis Vibratory Rate Gyroscope. Proc. Solid-State Sensors and Actuators, 1994.

[3] A. Shkel, R. Horowitz, A. Seshia, S. Park and R.T. Howe. Dynamics and Control of Micromachined Gyroscopes. American Control Conf., CA, 1999.

[4] S. Park and R. Horowitz. Adaptive Control for Z-Axis MEMS Gyroscopes. American Control Conference, Arlington, VA, June 2001.

[5] R.P. Leland. Adaptive Tuning for Vibrational Gyroscopes. Proceedings of IEEE Conference on Decision and Control, Orlando, FL, Dec. 2001.

[6] A. Shkel, R.T. Howe, and R. Horowitz. Modeling and Simulation of Micromachined Gyroscopes in the Presence of Imperfections. Int. Conf. on Modeling and Simulation of Microsystems, 1999.

[7] C. Acar, A. Shkel. Four Degrees-of-Freedom Micromachined Gyros. Journal of Modeling and Sim. of Microsystems, Vol. 2, pp. 71-82, 2001.

[8] J.A. Geen. A Path to Low Cost Gyroscopy. Solid-State Sensor and Actuator Workshop, Hilton-Head, SJ, 1998, pp. 51-54.

[9] W. Geiger, et.al. Decoupled Microgyros and the Design Principle DAVED. IEEE Sensors Journal, 2001, pp. 170-173.

[10] Y. Mochida, et.al. A Micromachined Vibrating Rate Gyroscope with Independent Beams for Drive and Detection Modes. Sensors and Actuators A, Vol. 80, 2000, pp. 170-178.

[11] M. Niu, et.al. Design and Characteristics of Two-Gimbals Micro-Gyroscopes Fabricated with Quasi-LIGA Process. Int. Conf. on Solid-State Sensor and Actuators, 1997, pp. 891-894.

[12] H.T. Lim, J.W. Song, J.G. Lee, and Y.K. Kim. A Few deg/hr Resolvable Low Noise Lateral Microgyroscope. Proceedings of IEEE MEMS Conference, NV, 2002, pp. 627-630.

[13] S.E. Alper, and T. Akin. A Symmetric Surface Micromachined Gyroscope with Decoupled Oscillation Modes. Sensors and Actuators A, Vol. 97, 2002, pp. 347-358.

[14] http://www.analogdevices.com.

[15] Y.S. Hong, J.H. Lee, and S.H. Kim. A Laterally Driven Symmetric Micro-Resonator for Gyroscopic Applications. Journal of Micromechanics and Microengineering, Vol. 10, 2000, pp. 452-458. 